\title{
On manifolds swept out by high dimensional quadrics
}

\author{
Mauro C. Beltrametti · Paltin Ionescu
}

Published online: 7 December 2007

C) Springer-Verlag 2007

\section{Erratum to: Math. Z. DOI 10.1007/s00209-007-0265-6}

We refer to our paper [1], using the same notation.

1. We thank C. Novelli and G. Occhetta for pointing out that in the statement of [1, Proposition 2.3] one needs to assume that $\mathcal{L}_{x}$ is irreducible if $x \in X$ is a general point. Then, the proof given in the paper is correct, with the following minor changes. First, the equality $F_{x}^{0}=F_{x}$ is true, as written. Next, from the irreducibility of $\mathcal{L}_{x}$, it follows that the closure of $\cup \mathcal{L}_{y}$, where $y \in F_{x}$ is a general point, is a well defined covering family of lines on $F_{x}$. So, we may apply, as claimed, [2, Proposition IV.3.13.3] to $F_{x}=F_{x}^{0}$. Note that, by [1, Proposition 2.1], whenever $\operatorname{dim}\left(\mathcal{L}_{x}\right) \geqslant \frac{n-1}{2}$, the family $\mathcal{L}_{x}$ is irreducible. Therefore, the rest of the paper is not affected.

2. We remark also that, given [1, Theorem 3.1], the statement of [1, Corollary 3.2] admits the following more general (and more natural) formulation:

Corollary In the hypothesis of [1, Theorem 3.1], assume moreover that, for two general points $x, x^{\prime} \in X$, the quadrics $Q_{x}$ and $Q_{x^{\prime}}$ intersect. Then $X$ is either a linear space or a hyperquadric.

The online version of the original article can be found under doi:10.1007/s00209-007-0265-6.

M. C. Beltrametti $(\bowtie) \cdot$ P. Ionescu

Dipartimento di Matematica, Università degli Studi di Genova, Via Dodecaneso, 35,

16146 Genova, Italy

e-mail: beltrame@dima.unige.it

P. Ionescu

e-mail: ionescu@dima.unige.it

P. Ionescu

University of Bucharest and Institute of Mathematics of the Romanian Academy, Bucharest, Romania 


\section{References}

1. Beltrametti, M.C., Ionescu, P.: On manifolds swept out by high dimensional quadrics. Math. Z. doi:10.1007/s00209-007-0265-6

2. Kollár, J.: Rational curves on algebraic varieties. Ergeb. Math. Grenzgeb. 32(3) (1996) 